\title{
Giant multicystic cystadenoma of Cowper's gland: a case report
}

Christian I. Villeda-Sandoval, Gustavo Romero-Vélez, Andrés Lisker-Cervantes, Mariano Sotomayor de Zavaleta, Alicia Trolle-Silva, Daniel Montante Montes de Oca, Ricardo Castillejos-Molina

Department of Urology (CIVS, GRV, ALC, MSZ, RCM) and Department of Pathology (ATS, DMMO), Instituto Nacional de Ciencias Médicas y Nutrición Salvador Zubirán, MEXICO

\section{ABSTRACT}

Main findings: We report what to our knowledge is the first case of a giant multicystic cystadenoma of the Cowper's glands. An otherwise healthy 41-year-old man presented with acute urinary retention. Physical examination showed a perineal mass. Different imaging techniques demonstrated a multicystic tumor and en bloc excision was performed. Histological evaluation showed that the tumor arised from the bulbourethral glands; immunohistochemistry proved positive staining for high molecular weight cytokeratin.

Case hypothesis: Cystic tumors in the pelvis can arise from different structures. Malignancy should be ruled out. Surgical excision can be diagnostic and curative.

Future implications: When evaluating a pelvic cystic tumor, Cowper's glands cystadenoma may be a differential diagnosis and must be considered. Similar to prostate cystadenomas, en bloc excision is the optimal treatment.

\section{ARTICLE INFO}

\section{Key words:}

Bulbourethral Glands; Cysts;

Cystadenoma

Int Braz J Urol. 2013; 39: 741-6

Submitted for publication:

May 05, 2013

Accepted after revision:

August 05, 2013

\section{CASE REPORT}

A 41-year-old man consulted his general physician complaining of dysuria and mild pain located in the hypogastric region. He had no other urinary tract symptom. His past medical and family history were unremarkable. He was diagnosed with a urinary tract infection (UTI) and received proper pharmacological treatment, which improved the symptoms.

Two months later, he presented with acute urinary retention, hence a transurethral catheter was placed. He was referred to the Urology clinic at our institution with a prostatic specific antigen (PSA) of $32 \mathrm{ng} / \mathrm{mL}(<4 \mathrm{ng} / \mathrm{mL})$. We did not consider this PSA levels to be reliable due to the recent manipulation of the urinary tract. Urinalysis revealed 22 leucocytes per high power field (pHPF), 12 red blood cells pHPF and positive nitrites. The transcatheter urine culture was positive for E. coli and K. pneumonia; both were sensitive to cefixime and he received $400 \mathrm{mg}$ qd for 10 days. The rest of the tests were within normal range values.

Physical examination revealed a non-tender perineal mass without signs of inflammation. During rectal examination a mobile tumor occluded the rectal lumen and was in continuity with the perineal mass. The prostate was not properly identified. The borders of the tumor were not reachable. No palpable mass was identified in abdominal examination. 
Transrectal ultrasound revealed a $13.4 \mathrm{x}$ $7.6 \times 10.4 \mathrm{~cm}$ tumor. It was described as a multicystic tumor with a small solid component. The cystic component represented $80 \%$ of the total volume; while the rest was described as solid. Magnetic Resonance Imaging (MRI) was performed and showed an 11 x 10 x $8 \mathrm{~cm}$ multicystic, hyperintense tumor with thick septa and solid nodules in T2 (Figure-1); both, septa and nodules enhanced with gadolinium. The coronal view showed a right urethral shift and cranial displacement of the bladder and the prostate. Seminal vesicles were distended and the prostate was in continuity with the tumor.
There was no evidence of lymph node involvement or free fluid in the pelvic or peritoneal cavity.

An incisional biopsy was not considered due to the Foley catheter dependence for bladder emptying and a tumor with an unknown invasive potential. Complete resection was considered to be most viable option to solve urinary retention and to define the diagnosis. As the tumor extended from the perineum to the pelvis, an open transperineal and retropubic approach was considered preoperatively (Figure-2).

Surgery was performed on December 18th, 2012. The tumor, the prostate and the seminal

Figure 1 - (A) MRI T2 Sagittal view (B) Macroscopic pathological specimen (C and D) MRI and macroscopic pathological specimen in colors. Prostate, seminal vesicles and the tumor are depicted in yellow, green and purple respectively.

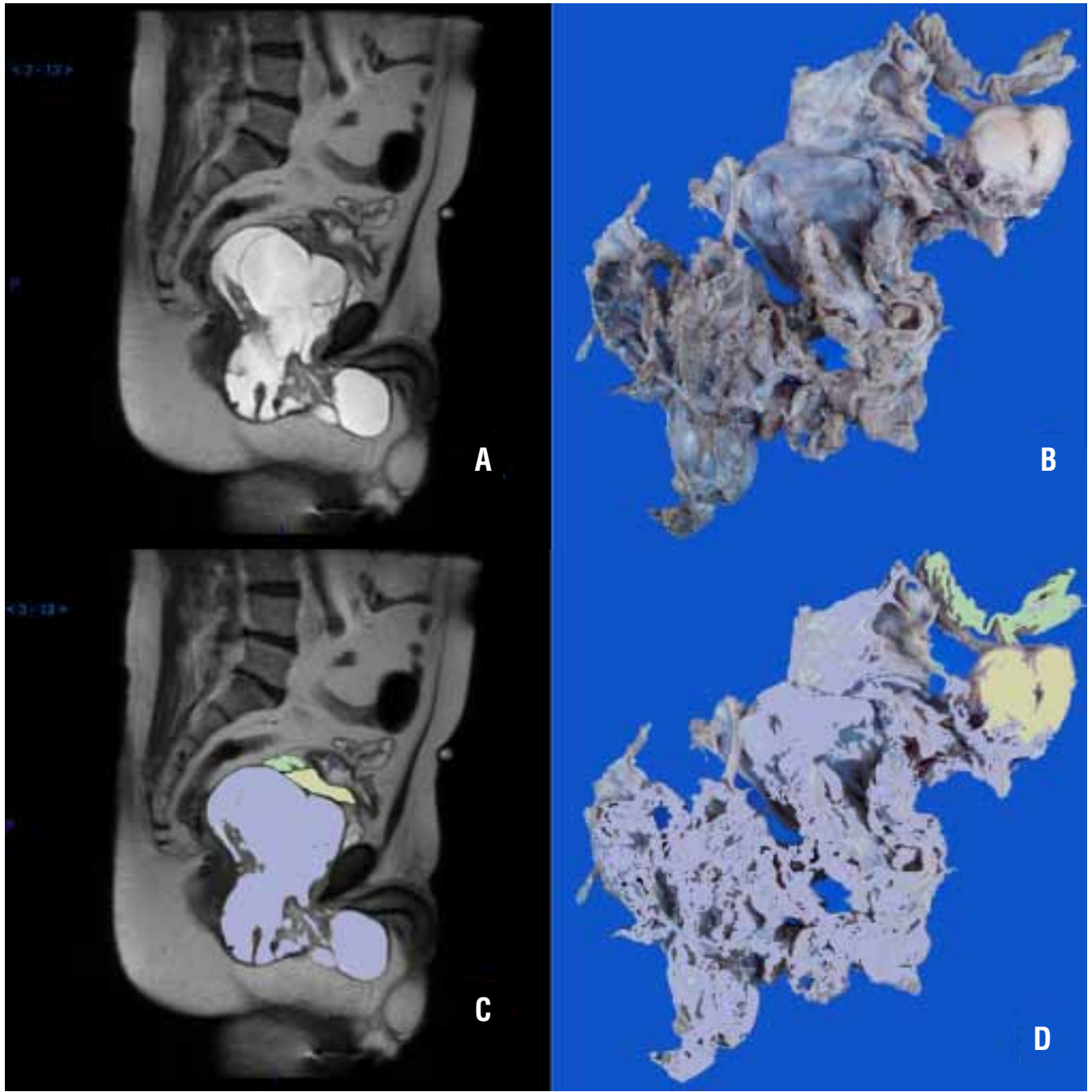


Figure 2 - Abdominal and perineal incisions.

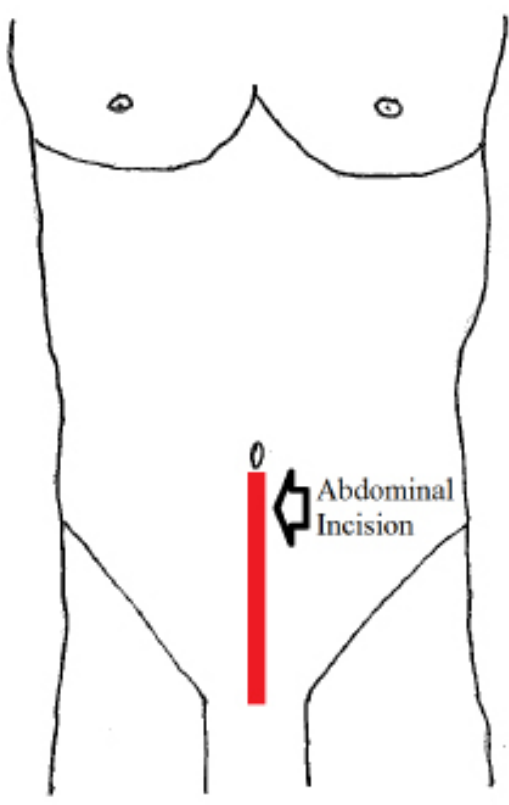

vesicles were removed en-bloc during the surgery. The anterior wall of the rectum was accidentally perforated and primarily repaired. Neurovascular bundles could not be preserved. A cystostomy and a protection colostomy were done. Significant bleeding $(4,000 \mathrm{~mL})$ occurred and 6 red blood cell packs were transfused. Vasopressor support was transiently employed for 24 hours. The patient stayed three days in the intensive care unit. Convalescence was uneventful and he was discharged 10 days after surgery.

A staff pathologist reviewed the specimen as our hospital does not count with a specialized uropathologist. It revealed a $20 \times 17 \mathrm{~cm}$ cystic tumor with a weight of $245 \mathrm{~g}$. The mass had smooth walls and contained a brownish liquid. Histologically, there were cystic dilatations of Cowper's glands alternating with normal glands. The cystic glands showed transition from a normal epithelium to a simple columnar epithelium (Figure-3). Immunochemistry staining was negative for PSA, while high molecular weight keratin (34betaE12) was positive (Figure-4). Lesions that have a negative PSA stain and positive staining for 34be-

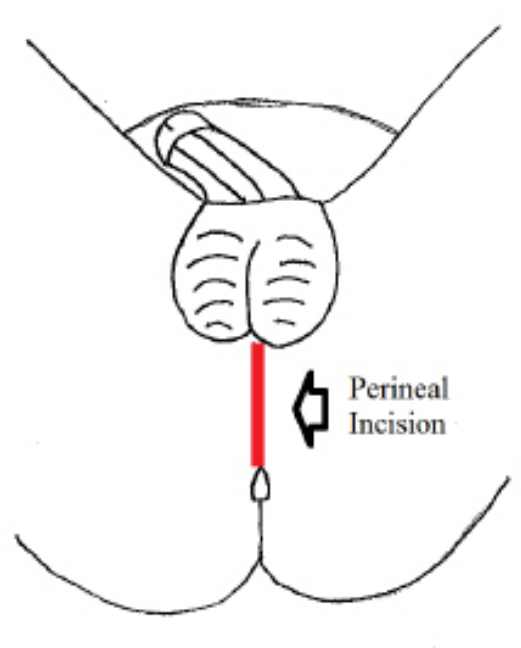

taE12 identify benign lesions of Cowper's glands, different from prostatic tissue $(1,2)$. Final diagnosis was giant multicystic adenoma of Cowper's gland. He has been in follow-up for four months without complaints or complications. Strict imaging follow-up was not performed due to the benign etiology of the tumor; he is scheduled for an abdominopelvic CT scan at 6 months follow-up. At that time we will decide if colonic anastomosis can be performed.

\section{CASE HYPOTHESIS AND RATIONAL}

Cystic lesions arising in the pelvis may originate from different structures. Phylloides tumor, simple prostate cyst, echinococcal cyst, closed syringocele, seminal vesicles cystadenoma, prostate cystadenoma and cystadenocarcinoma, are some of these lesions (3-7). Assessment with MRI and Computed Tomography (CT) scan can help identify possible etiologies; nevertheless the final diagnosis will be always dependent on final histopatological report. Bulbourethral glands origin was not suspected initially because this entity has not been described 
Figure 3 - (A) Hematoxylin and eosin. Cystic Cowper's glands, alternating with normal glands (10x); (B) Cystic dilation of Cowper's gland (10x); (C) High power view showing the transition from Cowper's gland normal epithelium to simple columnar epithelium lining the cystic portion (40x).

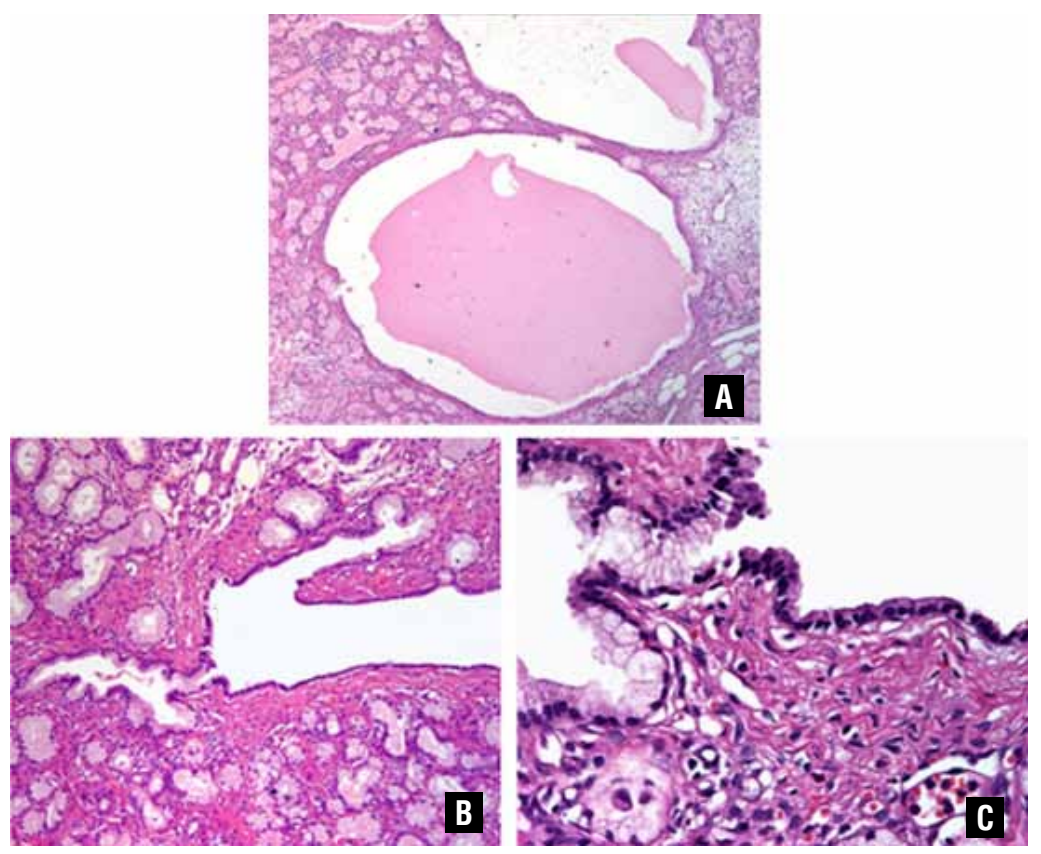

Figure 4 - Immunohistochemistry. (A) High molecular weight keratin (34betaE12) is expressed in normal and dilated glands (10x); (B) while PSA is negative in both (10x).

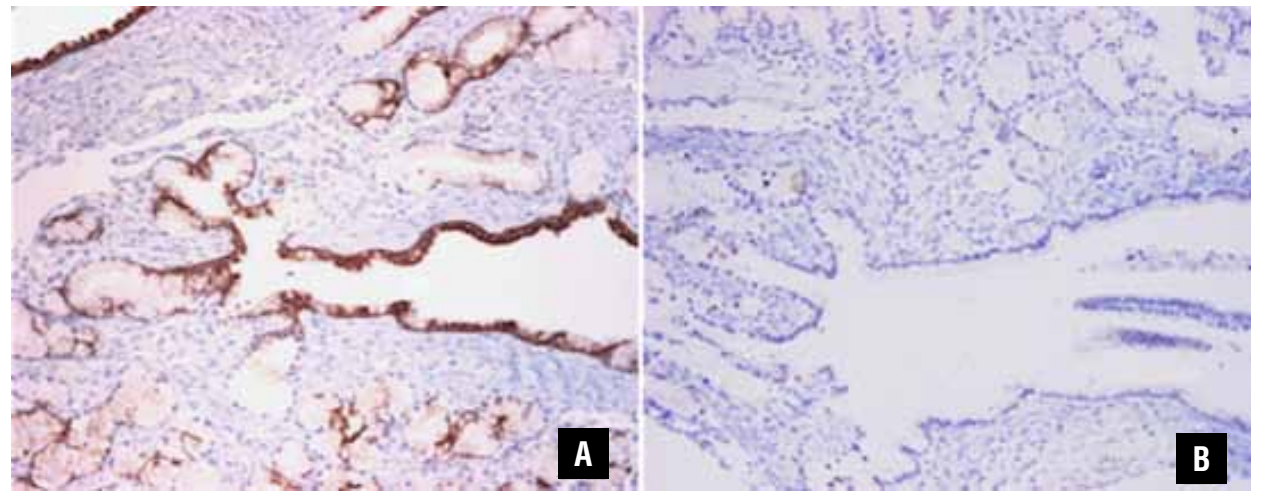

previously. However, this should be considered as a differential diagnosis in future cases presenting with cystic tumors in the pelvis.

\section{DISCUSSION}

We performed a PubMed search using MeSH terms for "Cystadenoma" and "Bulboure- thral glands". The query did not show any results. World Health Organization Pathology Classification and the Armed Forces Institute of Pathology were also consulted without findings; henceforth, to our knowledge this is the first case report of a cystadenoma arising from the Cowper's glands. Since there was no information about this tumor we extended the search for cystic lesions in the 
pelvis and tumors of the bulbourethral glands. Only articles in English and Spanish were included in the review.

Phylloides tumors of the prostate, also known as prostatic stromal proliferation of uncertain malignant potential are rare lesions with only 82 cases reported according to Bannowsky et al. (7). These tumors resemble those found in the mammary glands $(6,8)$. Obstructive symptoms or hematuria are usually found at presentation. PSA is slightly elevated in some reports while others show a normal value (7-9). Most of them present as cystic lesions that can be seen on CT scan and MRI but other presentations, such as polyps, have also been reported (8). Even though they can be classified as benign or malignant, multiple recurrences are frequently found on follow-up; so radical prostatectomy is warranted $(6,9)$. Immunostaining is helpful for diagnosis, being positive for CD34 and desmin (5-7).

Multicystic tumors of the prostate are rare and can be either cystadenomas or cystadenocarcinomas. Only 14 cystadenomas have been reported (10). Presentation can be with acute urinary retention, lower urinary tract symptoms, hematuria or even infertility $(10,11)$. The PSA is not always elevated (11); CT scan and MRI are useful techniques for diagnosis. Complete surgical excision of cystadenomas is the treatment of choice, with two recurrences reported $(4,5)$. Cystadenocarcinomas have a more aggressive nature, with higher local invasion and recurrence rates (4).

Cystic lesions can also arise from the bulbourethral glands; being the closed syringocele the most common (12). This is a congenital abnormality characterized by cystic dilation of the gland's duct. Presentation in adulthood is not common, they are usually asymptomatic but may present with lower urinary tract symptoms (1214). On imaging techniques (MRI) they appear as small cystic lesions (14); treatment consists of endoscopic incision. Tumors arising from these glands are extremely rare; only 20 cases of adenocarcinomas have been reported and only four had a cystic component (15-17).

To our knowledge, this is the first report of a Cowper's gland multicystic cystadenoma. As in prostatic benign cystic lesions, complete surgical excision is an appropriate treatment. There is not information about this entity; therefore all cases should be reported.

\section{ABBREVIATIONS}

UTI $=$ Urinary tract infection

PSA $=$ Prostatic Specific Antigen

HPF $=$ High power field

MRI = Magnetic Resonance Imaging

34betaE12 = High Molecular Weight Keratin

$\mathrm{CT}=$ Computed tomography.

\section{SEARCH PROTOCOL}

1. “cowper's glands"[Ti] AND ("tumours"[All Fields] OR "neoplasms"[MeSH Terms] OR "neoplasms"[All Fields] OR "tumors"[All Fields])

2. “cowper's glands"[Ti] AND ("neoplasms"[MeSH Terms] OR "neoplasms"[All Fields])

3. "cowper's glands"[Ti] AND ((“cysts”[MeSH Terms] OR "cysts"[All Fields] OR "cyst”[All Fields]) AND ("tumours"[All Fields] OR “neoplasms”[MeSH Terms] OR "neoplasms"[All Fields] OR "tumors"[All Fields]))

4. “cowper's glands"[Ti] AND ("neoplasms"[MeSH Terms] OR "neoplasms"[All Fields] OR “cancer”[All Fields])

5. “cowper's glands"[Ti] AND ("neoplasms"[MeSH Terms] OR "neoplasms"[All Fields] OR ("malignant"[All Fields] AND "tumors"[All Fields]) OR "malignant tumors"[All Fields])

6. ("bulbourethral glands"[MeSH Terms] OR ("bulbourethral"[All Fields] AND "glands"[All Fields]) OR "bulbourethral glands”[All Fields]) AND Cowper[All Fields]

7. ((“bulbourethral glands”[MeSH Terms] OR ("bulbourethral"[All Fields] AND "glands"[All Fields]) OR "bulbourethral 
glands"[All Fields]) AND Cowper[All Fields]) AND ("tumours"[All Fields] OR “neoplasms”[MeSH Terms] OR “neoplasms"[All Fields] OR "tumors"[All Fields])

\section{CONFLICT OF INTEREST}

None declared.

\section{REFERENCES}

1. Saboorian MH, Huffman H, Ashfaq R, Ayala AG, Ro JY: Distinguishing Cowper's glands from neoplastic and pseudoneoplastic lesions of prostate: immunohistochemical and ultrastructural studies.Am J Surg Pathol. 1997; 21: 1069-74.

2. Googe PB, McGinley KM, Fitzgibbon JF: Anticytokeratin antibody 34 beta E12 staining in prostate carcinoma. Am J Clin Pathol. 1997; 107: 219-23.

3. Damjanov I, Apic R: Cystadenoma of seminal vesicles. J Urol. 1974; 111: 808-9.

4. Tuziak T, Spiess PE, Abrahams NA, Wrona A, Tu SM, Czerniak B: Multilocular cystadenoma and cystadenocarcinoma of the prostate. Urol Oncol. 2007; 25: 19-25.

5. Levy DA, Gogate PA, Hampel N: Giant multilocular prostatic cystadenoma: a rare clinical entity and review of the literature. J Urol. 1993; 150: 1920-2.

6. Chung HC, Lee HS, Kim TI, Kim DI, Park KH, Song JM: A large cystic phyllodes tumor of the prostate. Yonsei Med J. 2009; 50: 174-6.

7. Bannowsky A, Probst A, Dunker H, Loch T: Rare and challenging tumor entity: phyllodes tumor of the prostate. J Oncol. 2009; 2009: 241270.

8. Latif Z, Clark D, Nairn ER, Gurun M: Phyllodes tumor of the prostate: a rare condition requiring careful surveillance. $\mathrm{J}$ Urol. 2003; 170: 1944.
9. Aguilar B, Villalobos M, Sotomayor M: Phyllodes tumor of the prostate. Urology. 2009; 73: 272.

10. Mosharafa AA, Torky MH, Ragab Y, Dahba N: Prostate cystadenoma presenting with obstructive azoospermia. J Androl. 2011; 32: 364-6.

11. Park JP, Cho NH, Oh YT, Choi YD: Giant multilocular prostatic cystadenoma presenting with obstructive aspermia. Yonsei Med J. 2007; 48: 554-6.

12. Chughtai B, Sawas A, O'Malley RL, Naik RR, Ali Khan S, Pentyala S: A neglected gland: a review of Cowper's gland. Int J Androl. 2005; 28: 74-7.

13. Selli C, Nesi G, Pellegrini G, Bartoletti R, Travaglini F, Rizzo M: Cowper's gland duct cyst in an adult male. Radiological and clinical aspects. Scand J Urol Nephrol. 1997; 31: 313-5.

14. Watson RA, Lassoff MA, Sawczuk IS, Thame C: Syringocele of Cowper's gland duct: an increasingly common rarity. J Urol. 2007; 178: 285.

15. Small JD, Albertsen PC, Graydon RJ, Ricci A Jr, Sardella WV: Adenoid cystic carcinoma of Cowper's gland. J Urol. 1992; 147: 699-701.

16. Trnski D, Custovic Z, Soric T, Ruzic B, Kruslin B: Primary adenoid cystic carcinoma arising in the region of Cowper's gland. BJU Int. 2003; 92 (Suppl 3): e66.

17. Hisamatsu H, Sakai H, Igawa T, Iseki M, Hayashi T, Kanetake $\mathrm{H}$ : Adenoid cystic carcinoma of Cowper's gland. BJU Int. 2003; 92(Suppl 3): e56-e57.

\author{
Correspondence address: \\ Dr. Ricardo Castillejos Molina \\ Instituto Nacional de Ciencias Médicas y \\ Nutrición Salvador Zubirán \\ Vasco de Quiroga \# 15 Col. Sección XVI \\ Tlalpan, CP 14000, México, DF \\ Fax: 54854380 \\ E-mail: rcastillejos@hotmail.com
}

